\title{
Parameter Selection of Oscillator Resonant Circuit Based on Stochastic Differential Equation
}

\author{
Yan Gang Feng ${ }^{1,2}$, Huang Xian $\mathrm{He}^{3} \&$ Xu Ning Jing ${ }^{1}$ \\ ${ }^{1}$ School of Electronic Information Engineering, Chengdu University, \\ No.1 Shiling Town, Chengdu, 610106, China \\ ${ }^{2}$ College of Computer Science, Sichuan University, No. 24 South Section 1, \\ Yihuan Road, Chengdu, 610065, China \\ ${ }^{3}$ School of Automation Engineering, University of Electronic Science and Technology \\ of China, No. 4, Section 2, North Jianshe Road, Chengdu, 611731, China \\ Email: uestcygf@163.com
}

\begin{abstract}
Because the choice of oscillator resonant circuit parameters depends mostly on experience, we propose nonlinear differential equations to describe an oscillator based on an equivalent circuit of the oscillator and then to describe the internal electrical noise of the oscillator by introducing a stochastic term that establishes a nonlinear stochastic differential equation to analyse the oscillator's behavior. For optimization of the oscillator resonant circuit parameters, first, we used Advanced Design System (simulation software of the Agilent company) to verify the conclusion, and then, using a $10 \mathrm{MHz}$ Pierce crystal oscillator, provided experimental evidence.
\end{abstract}

Keywords: circuit simulation; oscillators; parameter optimization; phase noise; resonant parameters; stochastic differential equation.

\section{Introduction}

The oscillator is one of the most important electronic devices in electronic circuits. It provides the reference frequency in many set-ups and is widely used in communications, electronics, telecommunications, aerospace, instruments and so on [1]. How to choose the parameters of a resonant circuit is of great significance to the oscillator characteristics and the oscillator frequency stability in oscillator circuits. Although the topic of noise in oscillators has engaged classical investigations of a qualitative nature [2,3], Leeson [4] was the first to propose a simple intuitive phenomenological model relating the level of phase noise in a widely used class of resonator-based oscillators to voltage and current noise sources in the circuit elements. Sauvage [5] used the mathematical principles involved to prove the validity of Leeson's model. This model has been widely embraced and serves well to predict oscillator phase noise induced by sources of white noise. However, while Leeson admits that device flicker (1/f) noise may determine the phase noise very close to the oscillation frequency, his model cannot explain why. Using a linear time variant (LTV)

Received May $4^{\text {th }}, 2013,1^{\text {st }}$ Revision February $13^{\text {th }}, 2014$, Accepted for publication March $4^{\text {th }}, 2014$. Copyright (C) 2014 Published by ITB Journal Publisher, ISSN: 2337-5779, DOI: 10.5614/j.eng.technol.sci.2014.46.2.4 
model for the oscillator, Hajimiri and Lee [6] have proposed a phase-noise analysis method that explains this up-conversion phenomenon, but it cannot predict the phase noise at frequency offsets very close to carrier. Kaertner [7], [8] and Demir [9] used the perspective of a state-space trajectory to analyze the phase noise of oscillators. In their works, the noise causing perturbation is decomposed into two parts. One part causes a deviation in the state-space solution along the unperturbed trajectory, effectually altering the phase of the solution. The other part results in a deviation that is considered an orbital perturbation. The orbital perturbation can be shown to remain small given a small noise. Subsequently, they analyzed the oscillator phase noise by a linearization of the oscillator equations around the noiseless periodic steadystate solution.

According to recent research, generally speaking there are two methods: direct analysis with a linear system or transferring the nonlinear system into the linear domain. Since the closed-loop gain of the oscillator has to be larger than the one at the beginning stage of oscillation, the output amplitude of the oscillator can be increased in order to amplify both the internal and the external noise of the oscillator [1]. When the output amplitude is increased to a certain value, the closed-loop gain will be decreased by the nonlinear characteristics of the active components in the oscillator. The signal of the oscillator can be stable only when the closed-loop gain of the oscillator is equal to one. Therefore, in order to make sure the oscillator can initialize the oscillation and maintain stable status, all oscillators have to be non-linear systems. Any linear process will definitely change the physical characteristics of the oscillator [1].

This paper directly describes oscillators using nonlinear autonomous differential equations, and introduces the noise signal as a term of a nonlinear autonomous differential equation. By setting up a nonlinear stochastic differential equation model, the phase noise of oscillators can be analysed. Using the widely used Pierce oscillator as an example, the modeling process illustrates the rationality of the model. Reference [10] uses this nonlinear stochastic differential equation model of the Pierce oscillator and a genetic algorithm to select the oscillator resonant circuit parameters, whose fitness function is based on the least phase noise. The results show that when the capacitance between the base and the emitter of the resonant circuit and the capacitance between the collector and the emitter of the resonant circuit are close, the phase noise can be better inhibited in the Pierce oscillator circuit. Their relationship is less than 2 to 8 times in reference [11]. In this research, Agilent's Advanced Design System simulation software was used to test this conclusion and subsequently an actual circuit was used to verify the conclusion. 


\section{Derivation of Model}

An ideal oscillator has the following output form:

$$
x=A_{0} \cos \left(2 \pi f t+\varphi_{0}\right)
$$

where $x$ is output of oscillator, $A_{0}$ is amplitude of oscillator, $f$ is output frequency of oscillator, $\varphi_{0}$ is initial phase, $A_{0}, f$ and $\varphi_{0}$ are all constant. The oscillators' output is a signal spectral line in the frequency domain of this case. Carefully observe Eq. (1), which must satisfy the following differential equation:

$$
\ddot{x}+(2 \pi f)^{2} x=0
$$

Considering the nonlinear essence of the oscillator, introduce nonlinear term $\varepsilon f(x, \dot{x})$ to describe the nonlinear active device of the oscillator. Without loss of generality, the oscillator without noise is described by the scalar, ordinary differential equation:

$$
\ddot{x}+x+\varepsilon f(x, \dot{x})=0
$$

where $\varepsilon$ is a real number. The function $f$ is nonlinear so $\varepsilon$ is a parameter that controls the degree of nonlinearity of the system. By introducing a noise term, we can get the equation to describe the oscillator with noise [12]

$$
\ddot{x}+x+\varepsilon f(x, \dot{x})=w(t)
$$

where $w(t)$ is the noise.

Considering the wide use of the Pierce oscillator, without loss of generality, the Pierce oscillator with grounded emitter is analyzed. The equivalent circuit schematic diagram is shown in Figure 1.

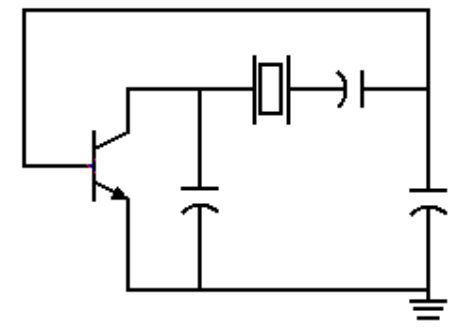

Figure 1 Pierce oscillator circuit with emitter grounded.

In order to facilitate the derivation, Figure 1 can be simplified as shown in Figure 2. 


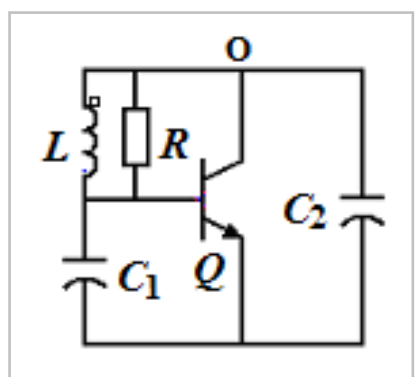

Figure 2 Pierce oscillator.

Established current equation for node o:

$$
I_{L}+I_{R}+I\left(V_{b c}\right)+C_{2} \frac{d V_{c}}{d t}=0
$$

where $I_{L}$ is the current of through inductor branch $L, I_{R}$ is the current of through resistance branch $R, I\left(V_{b c}\right)$ is the current of through the collector junction, $V_{b c}$ is the voltage at the two ends of the collector and the base. $V_{c}$ is the voltage of the collector terminal, considering:

$$
V_{b} \approx \frac{C_{2}}{C_{1}} V_{c}
$$

where $V_{b}$ is a voltage based terminal.

So Eq. (5) can be written as:

$$
\frac{\int\left(1-\frac{C_{2}}{C_{1}}\right) V_{c} d t}{L}+\left(1-\frac{C_{2}}{C_{1}}\right) \frac{V_{c}}{R}+I\left(V_{b c}\right)+C_{2} \frac{d V_{c}}{d t}=0
$$

Let:

$$
x=\int\left(1-\frac{C_{2}}{C_{1}}\right) V_{c} d t
$$

Then, substituting the above expression into Eq. (6), we obtain:

$$
\frac{C_{1} C_{2}}{C_{1}-C_{2}} \ddot{x}+\frac{x}{L}+\frac{\dot{x}}{R}+I_{S}\left(e^{\frac{\dot{x}}{V_{T}}}-1\right)=0
$$

where $I_{S}$ is the reverse saturation current of the PN junction, $V_{T}=k T / q$ is the thermal voltage, $k$ is the Boltzmann constant, $T$ is the absolute temperature, $q$ is the charge on the electron. 
Because the electric noise is the fundamental cause of oscillator phase jitter, the noise $w(t)$ is introduced to describe the electrical noise of the oscillator. The Pierce oscillator with noise equation can be described as follows:

$$
\frac{C_{1} C_{2}}{C_{1}-C_{2}} \ddot{x}+\frac{x}{L}+\frac{\dot{x}}{R}+I_{S}\left(e^{\frac{\dot{x}}{V_{T}}}-1\right)=w(t)
$$

Eq. (8) fits the form of the nonlinear stochastic differential equation model that is proposed in this paper, which shows the effectiveness of the model (4).

Reference [10] used this model, selecting the same intensity of white noise. The following conclusion was drawn: if the capacitance between the base and the emitter of the resonance circuit and the capacitance between the collector and the emitter of the resonance circuit are close, the phase noise can be better inhibited in the Pierce oscillator circuit.

\section{Simulation Verification}

In this research, we used Agilent's Advanced Design System 2008 [13] as simulation tool and a parallel type oscillator simulation circuit with grounded emitter to verify the conclusion. The simulation circuit was as shown in Figure 3.

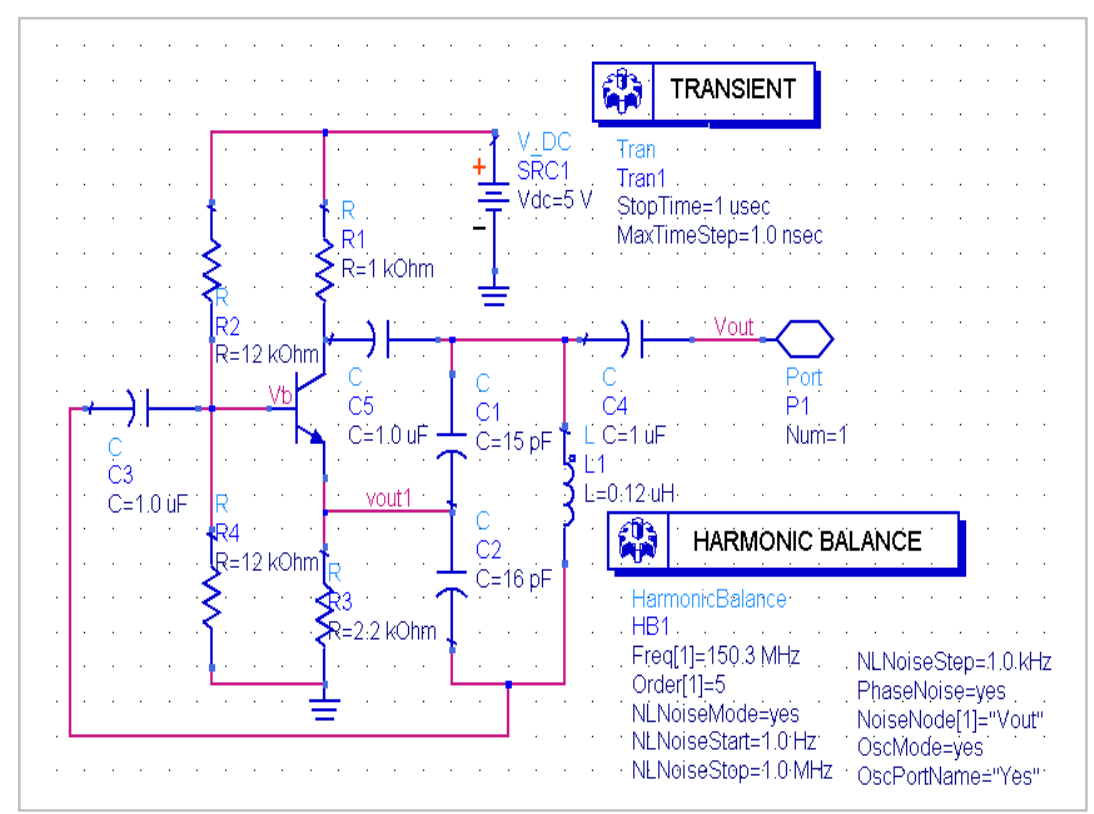

Figure 3 Parallel type oscillator simulation circuit with grounded emitter. 
Figure 4 is the simulation curve of the oscillation output and phase noise using empirical methods in this oscillation circuit for $\mathrm{C} 1=15 \mathrm{pF}, \mathrm{C} 2=30 \mathrm{pF}$.

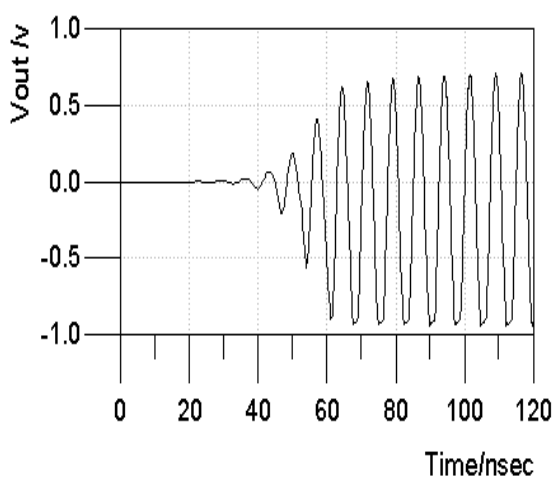

(a) Vout

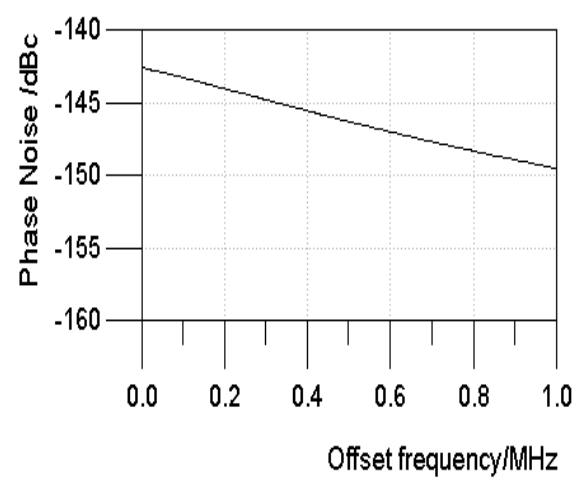

(b) Phase noise

Figure 4 The simulation curve of oscillation output and phase noise for $C_{1}=15 \mathrm{pF}, C_{2}=30 \mathrm{pF}$.

Figure 5 is the simulation curve of the oscillation output and phase noise using the conclusion of [10] in this oscillation circuit for $C_{1}=15 \mathrm{pF}, C_{2}=16 \mathrm{pF}$.

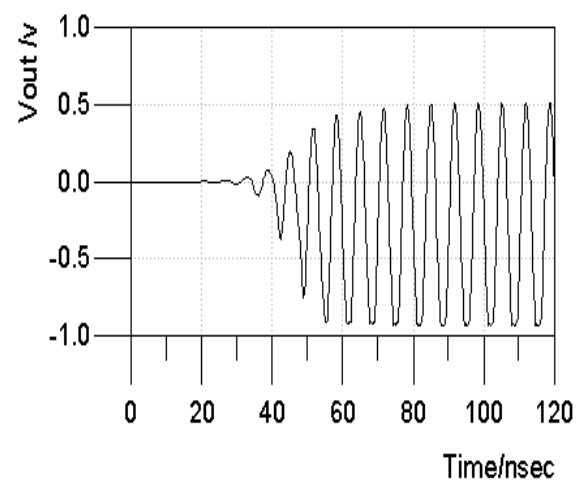

(a) Vout

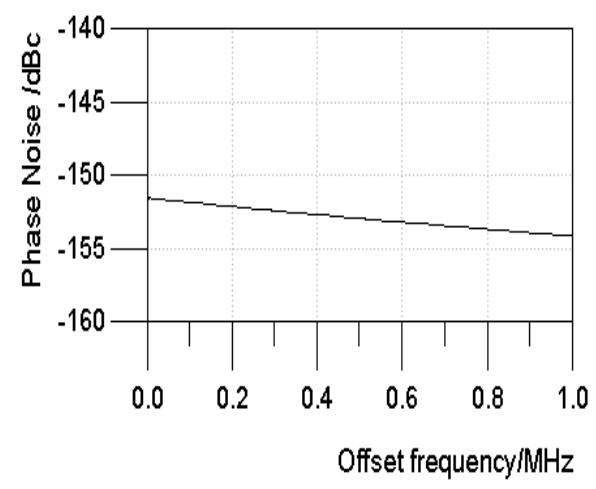

(b) Phase noise

Figure 5 The simulation curve of oscillation output and phase noise for $C_{1}=15 \mathrm{pF}, C_{2}=16 \mathrm{pF}$.

\section{Experimental Verification}

This research used an actual circuit to verify the conclusion. The equivalent circuit of the Pierce oscillator was as shown in Figure 6. 


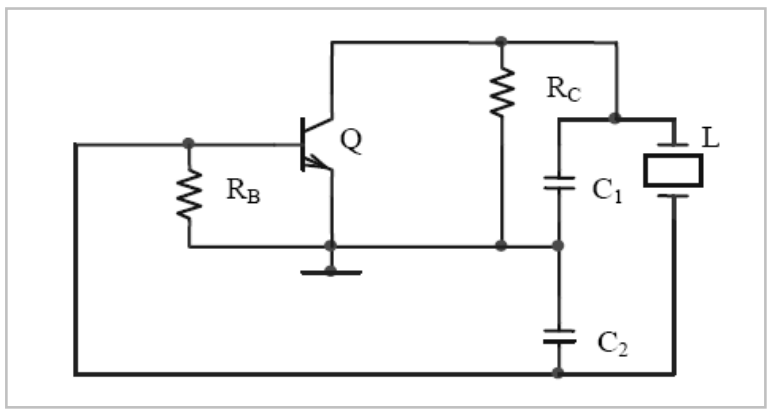

Figure 6 The Pierce equivalent circuit.

In this case, the design was a $10 \mathrm{MHz}$ Pierce crystal oscillator, while the other parameters of the oscillating circuit were the same. We only considered the relationship between the values of $\mathrm{C} 1$ and $\mathrm{C} 2$ to see how they influence the phase noise of the $10 \mathrm{MHz}$ Pierce crystal oscillator. The measurement instrument was an Agilent E5052B signal source analyzer.

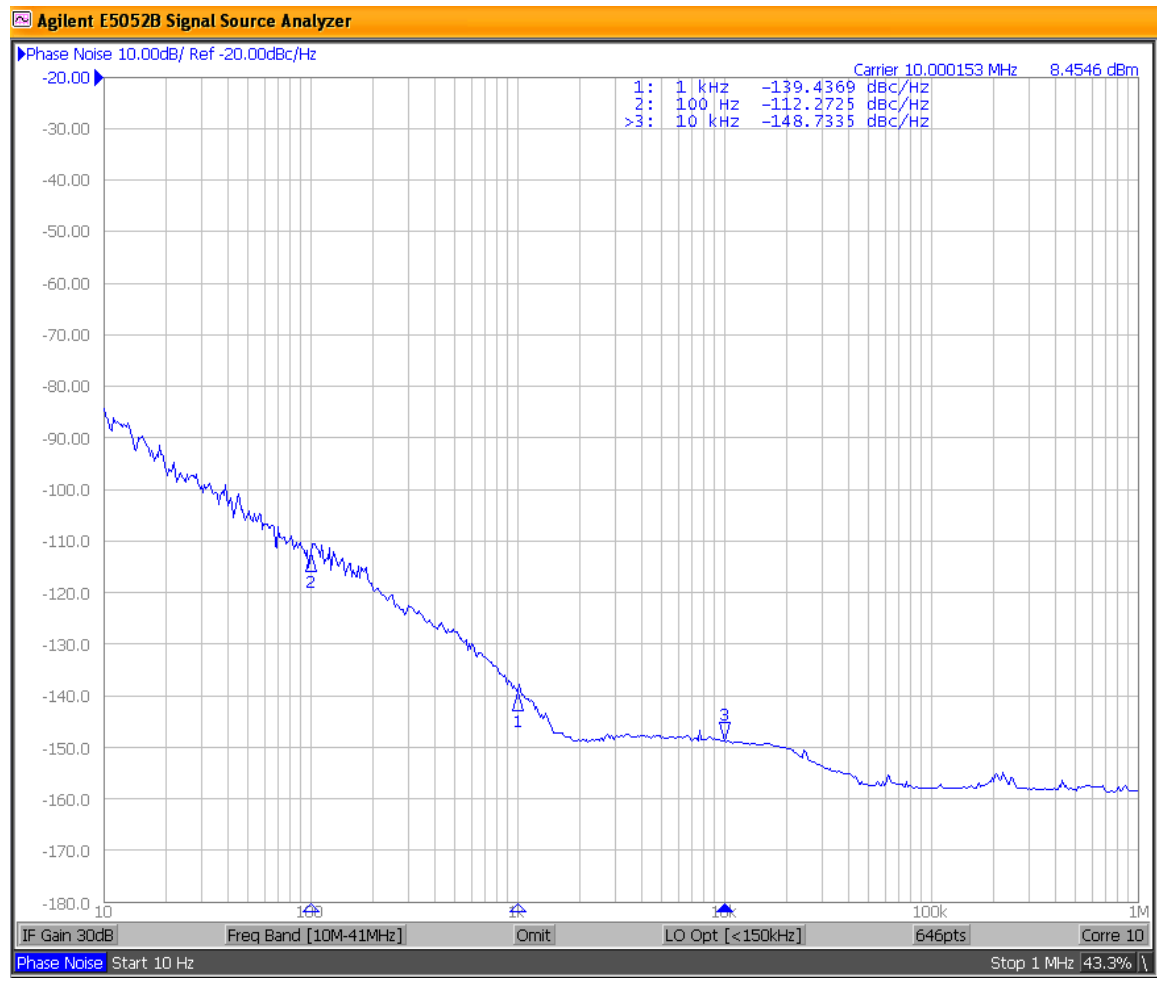

Figure 7 Test curve of the phase noise of $10 \mathrm{MHz}$ Pierce crystal oscillator with $\mathrm{C} 1=43 \mathrm{pF}, \mathrm{C} 2=100 \mathrm{pF}$. 
Figure 7 is the test curve of the phase noise of the $10 \mathrm{MHz}$ Pierce crystal oscillator for $\mathrm{C} 1=43 \mathrm{pF}, \mathrm{C} 2=100 \mathrm{pF}$. It shows that for deviations from the carrier frequency of $100 \mathrm{~Hz}, 1 \mathrm{kHz}, 10 \mathrm{kHz}$, the phase noise was -112.2725 dBc/Hz@100Hz,-139.4369 dBc/Hz@1kHz and -148.7335 dBc/Hz@10kHz.

According to the conclusion of [10], the phase noise can be reduced in a Pierce crystal oscillator when $C_{1}$ and $C_{2}$ are close. In the premise of meeting the feedback conditions, the test curve of the phase noise of the $10 \mathrm{MHz}$ Pierce crystal oscillator for $C_{1}=43 \mathrm{pF}, C_{2}=51 \mathrm{pF}$, as shown in Figure 8, shows that for deviations from the carrier frequency of $100 \mathrm{~Hz}, 1 \mathrm{kHz}, 10 \mathrm{kHz}$, the phase noise was -120.2828 dBc/Hz@100Hz, -145.4669 dBc/Hz@ $1 \mathrm{kHz}$ and -158.7771 $\mathrm{dBc} / \mathrm{Hz} @ 10 \mathrm{kHz}$.

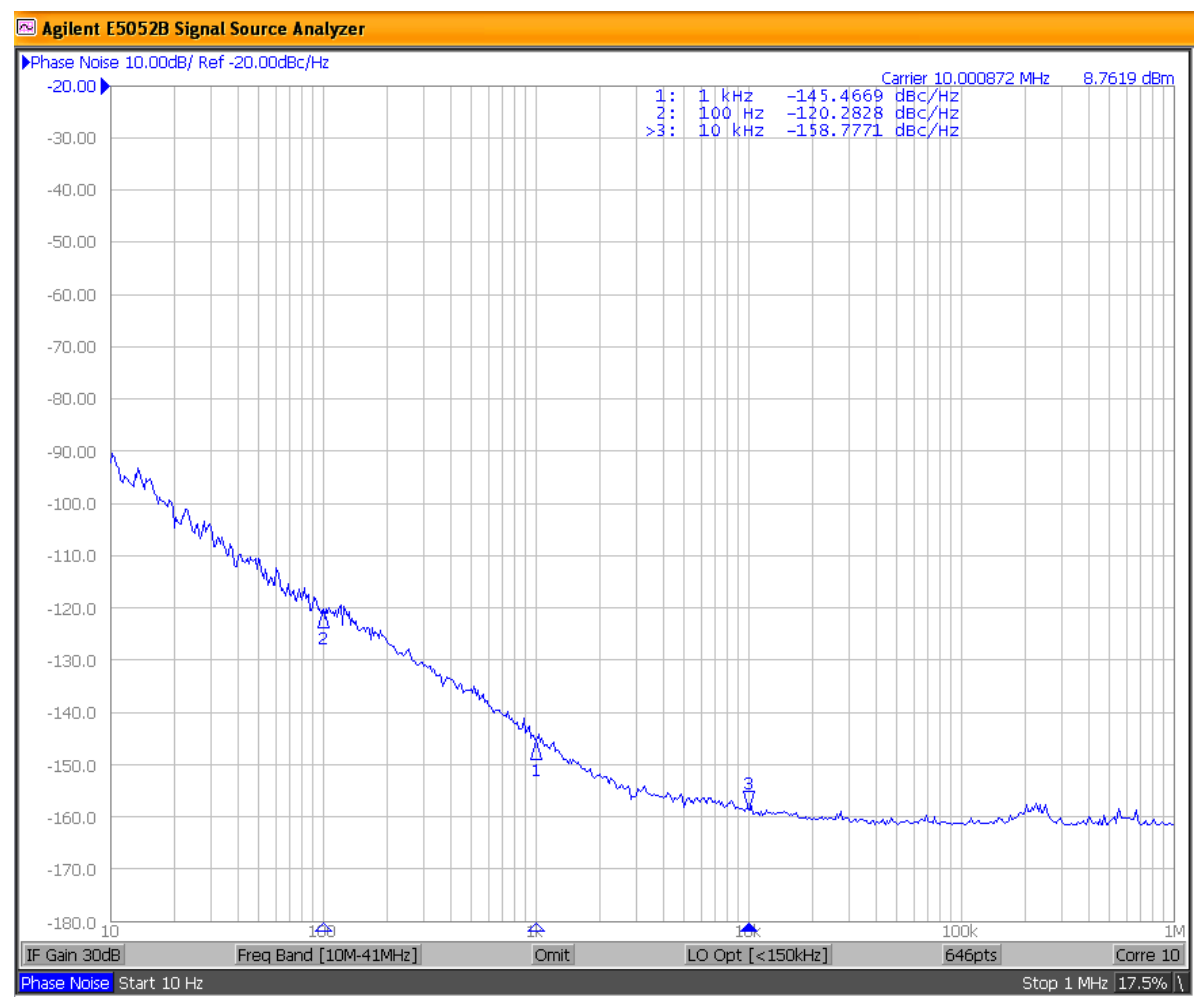

Figure 8 Test curve of the phase noise of $10 \mathrm{MHz}$ Pierce crystal oscillator for $\mathrm{C} 1=43 \mathrm{pF}, \mathrm{C} 2=51 \mathrm{pF}$.

\section{Conclusion}

Using nonlinear autonomous differential equations to describe an oscillator, the introduction of the noise signal as a term of nonlinear autonomous differential 
equations through the establishment of nonlinear stochastic differential equations corresponding to the analysis of the phase noise in oscillators is feasible. Comparing Figure 7 and Figure 8, we can see that the phase noise can be reduced in a Pierce crystal oscillator when the values of $\mathrm{C} 1$ and $\mathrm{C} 2$ are close. This conclusion was verified using an equivalent oscillator circuit of the Pierce crystal oscillator. In the resonant circuit, the capacitance between the base and the emitter and the capacitance between the collector and the emitter is less than 2 to 8 times the empirical relationship in reference [11].

Comparing the simulation results with the experimental results, although the simulation results were not completely consistent with the experimental results in numerical value (because the circuit simulation software is incapable of exactly simulating non-linear complex circuits), the above conclusion was confirmed. In fact, when $\mathrm{C} 1$ and $\mathrm{C} 2$ are close, the loaded $\mathrm{Q}$ will increase, which reduces the energy loss in the oscillation circuit - thus increasing the useful signal power - the signal-to-noise ratio is improved and therefore it can improve the phase noise. Comparing Figure 4 and Figure 5, we also found that when $\mathrm{C} 1$ and $\mathrm{C} 2$ are close, apart from inhibiting the phase noise in the Pierce oscillation circuit, also the starting oscillator characteristics are improved. This work provides empirical evidence for the optimization selection of oscillator resonant circuit parameters.

\section{Acknowledgments}

This work was supported by the National Natural Science Foundation of China (No. 11205022).

\section{References}

[1] Yan, G.F. \& Huang, X.H., Research on Phase Noise of Oscillators: A Survey, Journal of Astronautic Metrology and Measurement, 29(4), pp. 74-78, 2009.

[2] Edson, W.A., Noise in Oscillators, Proc. of IRE, 48(8), pp. 1454-1466, 1960.

[3] Mullen, J.A., Background Noise in Nonlinear Oscillators, Proc. of IRE. 48(8), pp. 1467-1473, 1960.

[4] Leeson, D.B., Simple Model of Feedback Oscillator Noise Spectrum, Proceedings Letters, IEEE, pp. 329-330, 1966.

[5] Sauvage, G., Phase Noise in Oscillators, A Mathematical Analysis of Leeson's Model, IEEE Transactions on Instrumentation and Measurement, 26(4), pp. 408-410, 1977.

[6] Hajimiri, A. \& Lee, T., A General Theory of Phase Noise in Electrical Oscillators, IEEE Solid State Circuits, 33 (2), pp. 179-194, 1998. 
[7] Kaertner, F.X., Determination of the Correlation Spectrum of Oscillators with Low Noise, IEEE Trans Microwave Theory Tech, 37(1), pp. 90-101, 1989.

[8] Kaertner, F.X., Analysis of White and $f-\alpha$ Noise in Oscillators, Int. J. Circuit Theory Appl., 18(5), pp. 485-519, 1990.

[9] Demir, A., Mehrotra, A. \& Roychowdhury, J., Phase Noise in Oscillators: A Unifying Theory and Numerical Methods for Characterization, IEEE Transactions on Circuits and Systems-I: Fundamental Theory and Applications, 47(5), pp. 655-674, 2000.

[10] Yan, G.F., Huang, X.H. \& Tan, H., The Optimal Selection of Oscillator Resonant Circuit Parameters Based on Genetic Algorithm, ACTA Metrologica Sinica, 31(2), pp. 165-169, 2010.

[11] Liu, M.L., Principle and Application of Oscillator, Higher Education Press, Beijing, pp. 41-43, 1984.

[12] Yan, G.F. \& Huang, X.H., Chaos on Phase Noise of Van Der Pol Oscillator, TELKOMNIKA, 8(3), pp. 301-308, 2010.

[13] Agilent Technologies, Advanced Design System 2001 User's Guide, 2001. 